\title{
Goshajinkigan oxaliplatin neurotoxicity evaluation (GONE): a phase 2, multicenter, randomized, double-blind, placebo-controlled trial of goshajinkigan to prevent oxaliplatin-induced neuropathy
}

\author{
Toru Kono - Taishi Hata - Satoshi Morita - Yoshinori Munemoto - Takanori Matsui • \\ Hiroshi Kojima · Hiroyoshi Takemoto • Mutsumi Fukunaga • Naoki Nagata • \\ Mitsuo Shimada $\cdot$ Junichi Sakamoto $\cdot$ Hideyuki Mishima
}

Received: 31 March 2013 / Accepted: 25 September 2013 / Published online: 12 October 2013

(c) The Author(s) 2013. This article is published with open access at Springerlink.com

\begin{abstract}
Purpose Oxaliplatin-induced peripheral neurotoxicity (OPN) is frequent and potentially severe, but successful treatment of this condition is still an unmet clinical need. We aimed to determine whether treatment with goshajinkigan (TJ-107), a traditional Japanese medicine, is better than placebo in preventing OPN in patients with advanced or recurrent colorectal cancer patients treated with standard FOLFOX regimens.

Methods In this phase 2, randomized, double-blind, placebo-controlled study, patients undergoing oxaliplatinbased chemotherapy were randomized to receive either oral
\end{abstract}

Findings from this study have been partially presented at the 36th European Multidisciplinary Cancer Congress (EMCC), September 23-27, 2011, Stockholm, Sweden; Multinational Association of Supportive Care in Cancer/International Symposium on Supportive Care in Cancer 2011 (MASCC/ISOO 2011), June 23-25, 2011, Athens, Greek; 13th World Congress on Gastrointestinal Cancer, June 22-25, 2011, Barcelona, Spain; and American Society of Clinical Oncology (ASCO) 47th Annual Meeting 2011, June 1-15, 2011, Chicago, USA.

Taishi Hata contributed equally as co-first author of this manuscript

T. Kono $(\bowtie)$

Advanced Surgery Center, Sapporo Higashi Tokushukai Hospital, Sapporo, 3-1, N33, E 14, Higashi-ku, Hokkaido 065-0033, Japan e-mail: kono@toru-kono.com

T. Hata

Department of Gastroenterological Surgery, Osaka University, Graduate School of Medicine, Suita, Osaka 565-0871, Japan

S. Morita

Department of Biostatistics and Epidemiology, Yokohama City University Medical Center, Yokohama, Kanagawa 232-0024, Japan
TJ-107 (7.5 g) or matching placebo daily. The severity of OPN was assessed according to the Common Toxicity Criteria for Adverse Events at baseline, every 2 weeks until the 8 th cycle, and every 4 weeks thereafter until the 26th week. The primary endpoint was the incidence of grade 2 or greater OPN until the 8th cycle of chemotherapy.

Results Analyses were done by intention to treat. Eightynine patients were randomly assigned to receive either TJ$107(n=44)$ or placebo $(n=45)$ between May 2009 and March 2010. The incidence of grade 2 or greater OPN until the 8 th cycle was 39 and $51 \%$ in the TJ-107 and placebo groups, respectively (relative risk (RR), 0.76; $95 \% \mathrm{CI}$, 0.47-1.21). The incidence of grade 3 OPN was $7 \%$ (TJ$107)$ vs. $13 \%$ (placebo) $(0.51,0.14-1.92)$. No concerns regarding toxicity emerged with TJ-107 treatment.

Conclusions TJ-107 appears to have an acceptable safety margin and a promising effect in delaying the onset of grade 2 or greater OPN without impairing FOLFOX efficacy.

Keywords Goshajinkigan · Peripheral neuropathy · Double-blind randomized trial · Oxaliplatin $\cdot$ Colorectal cancer

Y. Munemoto

Department of Surgery, Fukuiken Saiseikai Hospital,

Wadanaka-cho, Fukui 918-8503, Japan

T. Matsui $\cdot$ H. Kojima

Department of Surgery, Aichi Cancer Center, Aichi Hospital,

Okazaki, Aichi 444-0011, Japan

H. Takemoto $\cdot$ M. Fukunaga

Department of Surgery, Sakai Municipal Hospital, Sakai,

Osaka 590-0064, Japan 


\section{Introduction}

Oxaliplatin is considered one of the gold standard chemotherapeutic agents for advanced colorectal cancers and for adjuvant chemotherapy. However, oxaliplatin-induced peripheral neurotoxicity (OPN) is extremely common with the incidence varying from 82 to $98 \%$ [1-3]. Severe OPN occurs in 10 to $20 \%$ of patients [1, 4], and some may require dose reductions and discontinuation of treatment, potentially reducing the efficacy of chemotherapy and survival [5-7]. Despite considerable efforts to discover neuroprotective agents to prevent OPN [8], the best pharmacologic strategy for the management of OPN remains controversial [9-11].

In Japan, TJ-107 (goshajinkigan), a traditional Japanese medicine (Kampo) [12], has been frequently prescribed to alleviate symptoms of diabetic peripheral neuropathy such as numbness, cold sensation, and paresthesias/dysesthesias [13-15]. We hypothesized that TJ-107 might be effective against OPN and retrospectively investigated its use in a pilot study of 90 patients with advanced colorectal cancer undergoing FOLFOX therapy [16]. Patients were treated with TJ-107, calcium $(\mathrm{Ca})$ gluconate and magnesium (Mg) sulfate infusion, combination of TJ-107 and $\mathrm{Ca} /$ $\mathrm{Mg}$ infusion, or chemotherapy alone. Our results showed that the group receiving TJ-107 with FOLFOX regimen experienced significant improvement in OPN and showed a favorable safety profile. We subsequently conducted a small, single-arm prospective study in 45 patients who were treated with modified FOLFOX6 for advanced colorectal cancer, in which 22 patients receiving oral TJ-107 reported lower incidence of grades 2 and 3 OPN than that in the control group [17]. Hence, this phase 2, randomized, double-blind, placebo-controlled, exploratory trial was initiated to investigate the neuroprotective effect of TJ-107 for OPN.

N. Nagata

Department of Surgery, Kitakyushu General Hospital,

Kitakyushu, Fukuoka 800-0295, Japan

M. Shimada

Department of Surgery, Institute of Health Biosciences, The University of Tokushima Graduate School of Medicine,

Tokushima, Tokushima 770-8503, Japan

J. Sakamoto

Tokai Central Hospital, Kagamihara, Gifu 504-8601, Japan

H. Mishima

Unit of Cancer Center, Aichi Medical University, Nagakute, Aichi 480-1195, Japan

\section{Methods}

Study design

This was an exploratory, randomized, phase 2 trial to evaluate the efficacy of TJ-107 for preventing OPN in the Goshajinkigan Oxaliplatin Neurotoxicity Evaluation (GONE) study group conducted at 20 institutions in Japan [18].

\section{Eligibility criteria}

Patients were eligible if they had histologically confirmed colorectal cancer and were scheduled to undergo chemotherapy with infusional 5-fluorouracil (5-FU), leucovorin $(I-\mathrm{LV})$, and oxaliplatin (either FOLFOX4 or modified FOLFOX6 regimen). Patients had to have a good performance status (ECOG 0-1), adequate bone marrow function $\left(\mathrm{WBC} \geq 3,000\right.$ and $\leq 12,000 / \mathrm{mm}^{3}$, neutrophil count $\geq 1,500 / \mathrm{mm}^{3}$, platelet count $\geq 100,000 / \mathrm{mm}^{3}$ ), renal function (serum creatinine level less than the institutional upper limit of normal), and hepatic function (bilirubin $\leq 1.5$ times institutional normal, aspartate aminotransferase, and alanine aminotransferase levels less than 2.5 times the institutional upper limit of normal), life expectancy $\geq 12$ weeks, without evidence of clinical infection, and without preexisting peripheral neuropathy from any cause. Exclusion criteria included prior exposure to chemotherapy, except for oral fluorinated pyrimidine derivatives or 5-FU/l-LV in an adjuvant setting, use of other Kampo medicines, history of severe hypersensitivity (allergy) to any medications, other active malignancies or a history of other malignancies within the past 5 years, congestive heart failure, diabetes, or a history of a hemorrhagic stroke. Patients who were pregnant or nursing, taking a neuropathic pain medication, or receiving radiation were deemed ineligible.

This study was conducted in accordance with the Declaration of Helsinki and the Japanese Ministry of Health, Labour and Welfare guidelines, and informed consent was obtained from all participants. The study protocol was approved by the local Institutional Review Board at each participating institution.

\section{Randomization and masking}

Eligible patients were centrally randomized by a computergenerated allocation sequence in a 1:1 ratio to either TJ107 group or placebo group. Information regarding the necessary follow-up tests was then sent to the registration center at the non-profit organization Epidemiological and Clinical Research Information Network (ECRIN). Patients, investigators, and data collectors were all blinded to treatment allocation. 
Study medications

TJ-107 is a mixture of aqueous extracts from 10 crude herbs in fixed proportions (Rehmanniae Radix, $10.7 \%$; Achyranthis Radix, $6.4 \%$; Corni Fructus, $6.4 \%$; Moutan Cortex, $6.4 \%$; Alismatis Rhizome, $6.4 \%$; Dioscoreae Rhizome, $6.4 \%$; Plantaginis Semen, $6.4 \%$; Poria (Poria cocos Wolf), $6.4 \%$; processed Aconiti Tuber, $2.1 \%$; and Cinnamomi Cortex, $2.1 \%$ ). The extract powder of TJ-107 is commercially available in Japan and was obtained from Tsumura \& Co. (Tokyo, Japan). Matching placebo (Tsumura \& Co.) was specifically manufactured for this clinical trial. The appearance, color, and odor of the placebo were well controlled that both patients and clinicians were unable to distinguish this placebo from the original TJ-107. Dried powder $(2.5 \mathrm{~g})$ of TJ-107 or placebo was administered orally three times a day before each meal ( $7.5 \mathrm{~g} /$ day).

\section{Treatment}

Patients were randomly assigned to receive TJ-107 or placebo with either FOLFOX4 or mFOLFOX6 therapy. This treatment was initiated at the first delivery of FOLFOX and continued throughout the administration of chemotherapy and for 26 weeks beyond the completion of chemotherapy. Cycles of chemotherapy were given every 2 weeks until progressive disease or unacceptable toxicity occurred.

TJ-107 was given orally for 26 weeks starting on the day of oxaliplatin infusion. To avoid any possible influence on the assessment of neurotoxicity, $\mathrm{Ca} / \mathrm{Mg}$ infusion was prohibited only during the 26-week administration of TJ-107 and not throughout the chemotherapy regimen. FOLFOX4 therapy consisted of infusion of $l-\mathrm{LV}$ at $100 \mathrm{mg} / \mathrm{m}^{2}$ over $2 \mathrm{~h}$ followed by $5-\mathrm{FU}$ as a bolus $\left(400 \mathrm{mg} / \mathrm{m}^{2}\right)$ and a $22-\mathrm{h}$ infusion of 5 -FU $\left(600 \mathrm{mg} / \mathrm{m}^{2}\right)$ on day 1 and day 2, with infusion of oxaliplatin at $85 \mathrm{mg} / \mathrm{m}^{2}$ over $2 \mathrm{~h}$ on day 1 . This regimen was repeated every 2 weeks. mFOLFOX6 therapy consisted of infusion of $l-\mathrm{LV}$ at $200 \mathrm{mg} / \mathrm{m}^{2}$ over $2 \mathrm{~h}$ followed by $5-\mathrm{FU}$ as a bolus $\left(400 \mathrm{mg} / \mathrm{m}^{2}\right)$ and a $46-\mathrm{h}$ infusion of 5 -FU $\left(2,400 \mathrm{mg} / \mathrm{m}^{2}\right)$ with an infusion of oxaliplatin at $85 \mathrm{mg} / \mathrm{m}^{2}$ over $2 \mathrm{~h}$ on day 1 . This regimen was repeated every 2 weeks.

Adverse reactions including OPN were assessed at baseline (prior to starting FOLFOX + TJ-107 or FOLFOX + placebo), every 2 weeks until the 8th cycle and every 4 weeks thereafter until the 26th week according to the National Cancer Institute Common Terminology Criteria for Adverse Events (NCI-CTCAE v3.0). The severity of neurotoxicity was assessed by the study clinicians who were blinded to treatment allocation and used the sensory neuropathy items in NCI-CTCAE v3.0, which describe the four grades as follows: grade 1 , loss of deep tendon reflexes or paresthesia, including tingling, but not interfering with function; grade 2, objective sensory alteration or paresthesia, including tingling, interfering with function, but not interfering with activities of daily living (ADL); grade 3, sensory alteration or paresthesia interfering with ADL; and grade 4, permanent sensory losses that are disabling. In addition, patients rated their symptoms on a $0-4$ scale using the Functional Assessment of Cancer Therapy/Gynecological Oncology Group-Neurotoxicity (FACT/GOG-Ntx-12) score at screening, at baseline, and before each chemotherapy treatment. These subjective ratings were independently evaluated from the clinician-rated CTCAE grading. The follow-up period was 1 year after registration of the last patient.

\section{Statistical analysis}

The primary endpoint of this study was the incidence of grade 2 or greater OPN after 8 cycles of chemotherapy as assessed by the clinical investigators. The rate of incidence was compared between evaluable patients randomized to either the TJ-107 or the placebo group. The rate of occurrence of grade 2 or greater OPN was calculated for each group and compared using the chi-square test.

Secondary endpoints included the incidence and grading of OPN after each cycle, FACT/GOG-Ntx-12 score, time to occurrence of OPN, response rate to chemotherapy, and toxicity.

Previously, we found that the incidence of grade 2 or greater OPN was 15 and $45 \%$ (TJ-107 vs placebo) from the start of oxaliplatin treatment until the completion of cycle 8 [16]. On the basis of this data, we determined that in order to detect with $80 \%$ power while maintaining a significance level of $10 \%$ in a two-sided test, 35 patients per group would be required to compare the two treatment groups with a chi-square test. To account for possible dropouts, a minimum of 40 patients were enrolled in each group (80 in total). Randomization was achieved by using three strata: use of bevacizumab, the institution, and the presence of target lesions evaluated by Response Evaluation Criteria in Solid Tumors (RECIST) version 1.1. This trial is registered with the UMIN Clinical Trials Registry in Japan (UMIN000002211).

\section{Results}

A total of 93 patients were enrolled from May 1, 2009, to March 31, 2010. Of the 93 patients, 47 were assigned to the TJ-107 group and 46 to the placebo group. Four patients (3 receiving TJ-107 and 1 control) were withdrawn before initiation of treatment and included in the intention-to-treat set analysis (Fig. 1). The remaining 89 (96\%) patients were consequently assessable for efficacy 


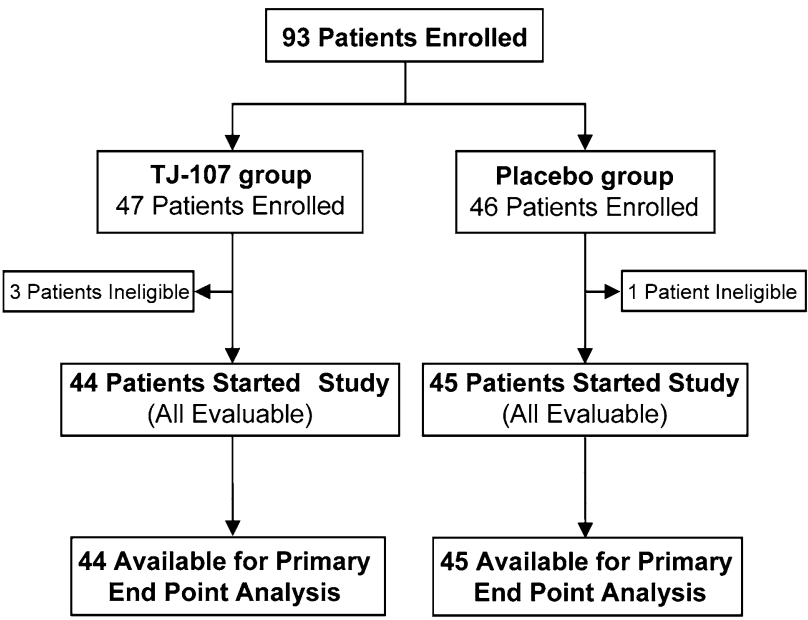

Fig. 1 CONSORT diagram

Table 1 Patient characteristics

\begin{tabular}{llll}
\hline & $\begin{array}{l}\text { TJ-107 } \\
(N=44)\end{array}$ & $\begin{array}{l}\text { Placebo } \\
(N=45)\end{array}$ & $P$ value \\
\hline Sex & & & \\
Male & 23 & 25 & 0.833 \\
Female & 21 & 20 & \\
Age & & 61 & 0.215 \\
Median & 67 & $36-82$ & \\
Range & $40-88$ & & 0.203 \\
Performance status & & 44 & \\
0 & 40 & 1 & 0.826 \\
1 & 4 & 30 & \\
Primary tumor & & 15 & 0.793 \\
Colon & 28 & 35 & \\
Rectum & 16 & 10 & \\
Chemotherapy & & & \\
First-line & 36 & 8 &
\end{tabular}

and toxicity. Reasons for premature withdrawal were disease progression (6 patients), adverse events (4), medical reasons (4), patient request (2), and complete response, operation, or death (1 each). Generally, demographic and background characteristics of the patients were well balanced between the TJ-107 group $(n=44)$ and placebo group $(n=45)$ ( $P$ values ranging from $0.203-0.833$ ) (Table 1).

Incidence of OPN

Data on the incidence of grade 2 or greater OPN and grade 3 OPN until the 8th cycle are summarized (Table 2). The incidence of grade 2 or greater OPN until
Table 2 Oxaliplatin-induced peripheral neurotoxicity until the 8th cycle

\begin{tabular}{llll}
\hline \multicolumn{4}{l}{ Oxaliplatin-induced peripheral neurotoxicity until the 8th cycle } \\
\hline & $\begin{array}{l}\text { TJ-107 } \\
(N=44)(\%)\end{array}$ & $\begin{array}{l}\text { Placebo } \\
(N=45)(\%)\end{array}$ & Relative risk [95 \%CI] \\
\hline Grade $\geq 2$ & 39 & 51 & $0.76[0.47-1.21]$ \\
Grade 3 & 7 & 13 & $0.51[0.14-1.92]$ \\
\hline
\end{tabular}

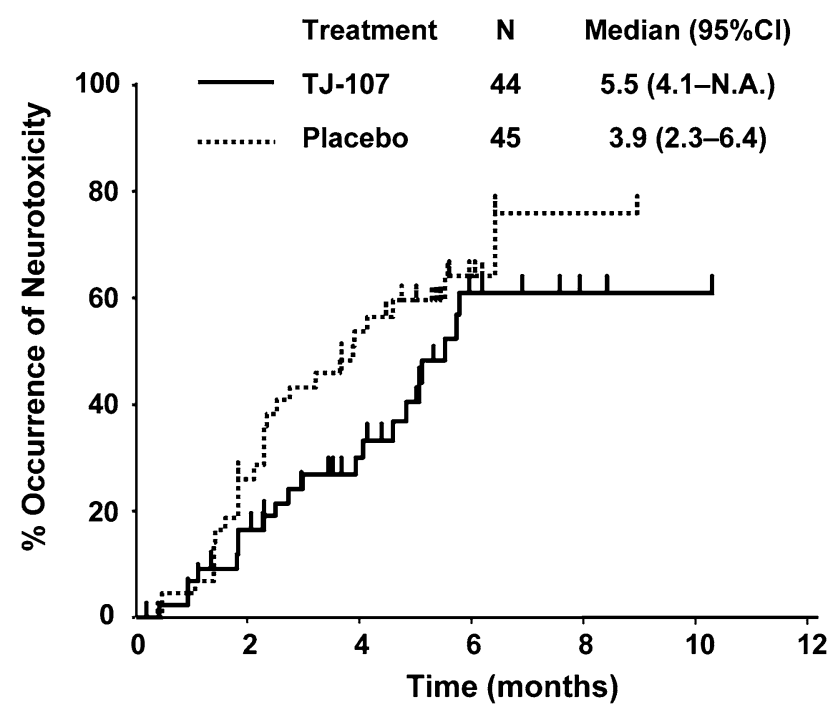

Fig. 2 Time to occurrence of grade 2 or greater neurotoxicity. Solid line TJ-107, broken line Placebo

the 8th cycle was $39 \%$ in the TJ-107 group and $51 \%$ in the placebo group (relative risk (RR) 0.76; $95 \%$ confidence interval (CI) 0.47-1.21). Similarly, the incidence of grade 3 OPN until the 8th cycle was $7 \%$ in the TJ-107 group and $13 \%$ in the placebo group (RR 0.51; $95 \% \mathrm{CI}$ 0.14-1.92).

The time to occurrence of grade 2 or greater OPN is shown in Fig. 2. In patients who developed grade 2 or greater OPN, the median time to occurrence was 5.5 months (95\%CI 4.1-N.A.) in the TJ-107 group and 3.9 months (95\% CI 2.3-6.4) in the placebo group (RR $0.65 ; 95 \%$ CI $0.36-1.17)$. The median time to occurrence of grade 3 neurotoxicity was better controlled in the TJ-107 group (RR 0.71; $95 \%$ CI 0.29-1.77). The median frequency of occurrence of OPN at 26 weeks was 54.1 and $62.5 \%$ (RR 0.86) in the TJ-107 and placebo groups, respectively.

When we stratified the patients based on FOLFOX regimen (i.e., FOLFOX4 vs. mFOLFOX6) and performed a subanalysis to determine whether there was a difference in the effects of TJ-107 on the occurrence of OPN, we found that there was no significant difference between 
Table 3 Median FACT/GOG-Ntx-12 score

\begin{tabular}{llcc}
\hline & $\begin{array}{l}\text { TJ-107 } \\
(N=44)\end{array}$ & $\begin{array}{l}\text { Placebo } \\
(N=45)\end{array}$ & $P$ value \\
\hline 8 weeks & 6.0 & 9.0 & 0.421 \\
26 weeks & 7.0 & 10.5 & 0.151 \\
\hline
\end{tabular}

* FACT/GOG-Ntx-12: Functional Assessment of Cancer Therapy/ Gynecological Oncology Group-Neurotoxicity-12 score

Table 4 Tumor response rate

\begin{tabular}{lccc}
\hline Overall & $\begin{array}{l}\text { TJ-107 } \\
(N=27)\end{array}$ & $\begin{array}{l}\text { Placebo } \\
(N=23)\end{array}$ & $P$ value \\
\hline Complete response (CR) & 1 & 1 & \\
Partial response (PR) & 14 & 10 & \\
Stable disease (SD) & 9 & 11 & \\
Progression disease (PD) & 3 & 1 & \\
Not evaluable (NE) & 0 & 0 & \\
CP + PR & $15(56 \%)$ & $11(48 \%)$ & 0.777 \\
$95 \%$ CI & $0.37-0.74$ & $0.27-0.68$ & \\
CR + PR + SD & $24(89 \%)$ & $22(96 \%)$ & 0.614 \\
$95 \%$ CI & $0.77-1.00$ & $0.87-1.00$ & \\
\hline
\end{tabular}

treatment groups albeit the small sample size in each group.

The median FACT scores of the TJ-107 and placebo groups were 6.0 vs. $9.0(P=0.421)$ at 8 weeks and 7.0 vs. $10.5(P=0.151)$ at 26 weeks (Table 3$)$. Although the differences were statistically unremarkable, patients receiving TJ-107 tended to show milder symptoms of neurotoxicity than those who received placebo.

\section{Tumor response rate}

The anti-tumor effect was assessed in 27 (TJ-107) and 23 (placebo) patients who had a target lesion at the time of enrollment. Bevacizumab was administered in $74 \%$ (20/27) and $74 \%(17 / 23)$ of patients in the TJ-107 group and the placebo group, respectively (Table 4).

The overall chemotherapy response rates were $56 \%$ in the TJ-107 group and $48 \%$ in the placebo group. In addition, $89 \%$ (TJ-107) and $96 \%$ (placebo) of patients demonstrated disease control (complete response, partial response, or stable disease) (Table 4).

\section{Toxicity assessment}

TJ-107 used in this study appeared to be well tolerated. There were no significant differences between the two groups in terms of toxicity. The most common adverse events likely caused by the chemotherapy were anorexia,
Table 5 Incidence of adverse events

\begin{tabular}{lcll}
\hline All grades & & & \\
\hline & $\begin{array}{l}\text { TJ-107 }(N=44) \\
(\%)\end{array}$ & $\begin{array}{l}\text { Placebo }(N=45) \\
(\%)\end{array}$ & $P$ value \\
\hline Fatigue & $25(57)$ & $26(58)$ & 1.000 \\
Anorexia & $27(61)$ & $22(49)$ & 0.289 \\
Nausea & $20(45)$ & $28(62)$ & 0.139 \\
Vomiting & $4(9)$ & $13(29)$ & 0.029 \\
Stomatitis & $19(43)$ & $16(36)$ & 0.519 \\
Diarrhea & $15(34)$ & $10(22)$ & 0.245 \\
Hand-food & $11(25)$ & $7(16)$ & 0.302 \\
$\quad$ syndrome & & & 0.230 \\
Allergic reaction & $8(18)$ & $4(9)$ & 0.494 \\
Febrile & $0(0)$ & $2(4)$ & 1.000 \\
$\quad$ neutropenia & & $2(4)$ & 0.494 \\
Constipation & $2(5)$ & $2(4)$ & 1.000 \\
Ileus & $0(0)$ & $4(9)$ & 0.052 \\
Total Bilirubin & $4(9)$ & $23(51)$ & 0.070 \\
AST & $13(30)$ & $19(42)$ & 0.271 \\
ALT & $10(23)$ & $19(42)$ & \\
ALP & $13(30)$ & & \\
\hline
\end{tabular}

Table 6 Incidence of hematologic toxicity events

\begin{tabular}{lclc}
\hline & $\begin{array}{l}\text { TJ-107 } \\
(N=44)(\%)\end{array}$ & $\begin{array}{l}\text { Placebo } \\
(N=45)(\%)\end{array}$ & $P$ value \\
\hline All grades & & & \\
Leukopenia & $21(48)$ & $27(60)$ & 0.291 \\
Neutropenia & $15(34)$ & $21(47)$ & 0.282 \\
Anemia & $30(68)$ & $31(69)$ & 1.000 \\
Thrombocytopenia & $12(27)$ & $15(33)$ & 0.646 \\
Grade $\geq 3$ & & & \\
Leukopenia & $1(2)$ & $2(4)$ & 1.000 \\
Neutropenia & $10(23)$ & $15(33)$ & 0.347 \\
Anemia & $1(2)$ & $1(2)$ & 1.000 \\
Thrombocytopenia & $0(0)$ & $0(0)$ & 1.000 \\
\hline
\end{tabular}

fatigue, nausea, and stomatitis, which were reported at similar rates from patients of both groups (Table 5). Vomiting was significantly suppressed in patients on TJ-107 compared with controls ( 9 vs. $29 \%, P=0.029$ ). In the context of systemic chemotherapy, most of these events were likely related to chemotherapy-induced toxicity, yet none of them were considered TJ-107 related. Eighteen hematologic toxicity events of grade 3 or greater (15 neutropenia) in the placebo group were reported, and 12 events (10 neutropenia) were noted in the TJ-107 group (Table 6). One patient in the placebo group died as a direct result of progressive disease. 


\section{Discussion}

This is the first placebo-controlled, randomized, exploratory study that assessed the efficacy of oral TJ-107 in the treatment of OPN in colorectal cancer patients undergoing oxaliplatin-based chemotherapy.

Our randomized, phase 2, exploratory trial using a placebo was designed to assess the potential success of oral TJ-107 in the phase 3 setting, rather than provide solid data on its efficacy. Results of this study showed promising relative risk that support the clinical activity of oral TJ-107 against OPN, particularly acute cold-associated neuropathy, in patients who received FOLFOX therapy (FOLFOX4 or mFOLFOX6) for colorectal cancer without imposing negative impact on oxaliplatin-based anti-tumor effect. Additionally, TJ-107 did not cause any adverse effects during the trial. Our findings, which showed improvement in both CTCAE grades and patient-rated FACT/GOG-Ntx scores, suggest that TJ-107 delays the occurrence of grade 2 or greater OPN during active treatment although its therapeutic effect may plateau after 6.5 months of continuous administration and that the development of neurotoxicity was not correlated with the completion of oxaliplatin treatment. Given the difficulty of generating statistically robust data with a small sample size, we surmised that our data warrant further investigation in a large phase 3 setting.

As an exploratory, phase 2 trial of patients with advanced or relapsed colorectal cancer (some with unresectable cancer), we conducted the endpoint assessment after 8 cycles rather than at treatment completion on the basis of a previous study that reported the high likelihood of detecting the side effects of oxaliplatin after 8 cycles [19], suggesting that this time point may be critical for deciding whether to continue oxaliplatin-based chemotherapy. Furthermore, a postmarketing drug surveillance of TJ-107 in Japan has shown that the median time to occurrence of grade 3 neuropathy with motor disorder was after 8 cycles. Taken together, these data suggest the evaluation of neuropathy at its peak incidence after 8 cycles to be clinically more meaningful than delaying the evaluation until treatment completion.

TJ-107 is a complex drug containing 10 medicinal herbs with a wide spectrum of pharmacologic actions [16]. Experimental studies have shown that TJ-107 relieves neurologic symptoms of diabetic peripheral neuropathy such as cold hyperalgesia and mechanical allodynia [13-15] primarily by the action of its analgesic component, detoxified Aconiti Tuber. The purported mechanisms by which this component works in concert with the other components of TJ-107 to exert a neuroprotective effect include (1) evoking the release of dynorphin and activating endogenous $\kappa$-opioid receptors to improve numbness or paresthesia [20, 21], (2) decreasing the release of transmitter proteins and sensory receptors associated with C-fiber nociceptor activation $[22,23]$, and (3) promoting nitric oxide production to improve blood supply to the nerves [24]. Furthermore, a recent experimental study has demonstrated that TJ-107 ameliorates the pain associated with OPN in rats without affecting the anti-tumor activity of oxaliplatin [25], which is in line with our findings. Interestingly, we also found that TJ-107 significantly decreased vomiting compared with placebo. The precise mechanism remains unclear but one of the components of TJ-107, Poria (Poria cocos Wolf), has shown an antiemetic effect through 5-HT3A receptor inhibition [26, 27].

According to the Multicenter International Study of Oxaliplatin, 5-Fluorouracil and Leucovorin in the Adjuvant Treatment of Colon Cancer (MOSAIC) study, the reported incidences of OPN were as follows: grade 1 (48 \%), grade $2(32 \%)$, and grade $3(12 \%)$ [1]. In the present study, the incidence of grade 2 and grade 3 OPN in the placebo group until the 8th cycle was $51 \%$ and $13 \%$, respectively, which is consistent with the MOSAIC study [1]. There was no marked difference in time to treatment failure in this study. One possible explanation for this is that even if greater than grade 2 OPN had occurred once, oxaliplatin administration was continued so long as OPN was downgraded to grade 2 on the day of administration. Another possibility is that patients may not acknowledge or report neuropathic symptoms for fear of missing out on an effective cancer treatment. Thus, it seems imperative to discover an agent that has sufficient evidence to decrease OPN development.

Many agents have been tested, in both humans and experimental animals, to ameliorate OPN [28]. Recently, the antidepressant drug venlafaxine, which is also used to manage pain associated with diabetic peripheral neuropathy, has been reported to significantly decrease the incidence of acute OPN in a placebo-controlled, randomized, phase 3 trial; however, grade 1-2 vomiting was observed more frequently in patients who received venlafaxine [29]. TJ-107 has also been used to treat painful diabetic peripheral neuropathy [13-15], but our study showed that TJ-107 significantly decreased vomiting compared to placebo, and other common adverse events due to chemotherapy did not worsen with TJ-107 treatment. Effective preventive treatments must not only mitigate neurotoxicity but must also preserve the antineoplastic effect of chemotherapeutic drugs. In this study, no between-group differences were found in response rates to chemotherapy or in the survival rates, suggesting that TJ-107 had no influence on FOLFOX therapy. Moreover, TJ-107 is an easily administered alternative that does not produce serious adverse effect, rendering it conducive to increasing compliance among patients and health care practitioners in a cancer treatment setting.

This is the first, randomized, phase 2, exploratory trial of TJ-107 whose study design itself is comparable to that 
of an older phase 3 trial design. The primary objective of this phase 2 trial study was to determine whether our findings would warrant validation in a phase 3 setting and not necessarily to obtain concrete data that show statistical significance. In other words, our aim was to define the characteristics of TJ-107 against OPN relative to placebo in order to refine the design of a phase 3 trial. Through this study, we were able to obtain a more accurate estimation of sample size and confirm that TJ-107 was similar to placebo in terms of toxicity despite the small sample size, and TJ107 prevented the progression and development of severe neurotoxicity, one of the primary dose-limiting factors of oxaliplatin-based chemotherapy. Taken together, our findings suggest that this trial served as an effective platform for testing the efficacy of a novel agent like TJ-107 in oncology and for designing and accelerating the transition from phase 2 to a large phase 3 trial that employs objective measures.

\section{Conclusions}

Findings from this phase 2, exploratory trial suggest that oral TJ-107 has acceptable margins of safety and tolerability and a promising effect in delaying the onset of grade 2 or greater OPN in colorectal cancer patients treated with oxaliplatin.

\begin{abstract}
Acknowledgments The authors thank the patients and all investigators and research support staff at the participating centers. We would also like to thank the trial management group, independent data monitoring and trial steering committees for overseeing the trial. We also thank Chigusa Abe for her excellent work as the principle research coordinator. Funding was partially provided by Epidemiological and Clinical Research Information Network (ECRIN). ECRIN provided peer-reviewed approval for the trial but had no other role in study design, collection, analysis, interpretation of data, or writing of the report.
\end{abstract}

\section{Conflict of interest None.}

Open Access This article is distributed under the terms of the Creative Commons Attribution License which permits any use, distribution, and reproduction in any medium, provided the original author(s) and the source are credited.

\section{References}

1. André T, Boni C, Mounedji-Boudiaf L, Navarro M, Tabernero J, Hickish T, Topham C, Zaninelli M, Clingan P, Bridgewater J, Tabah-Fisch I, de Gramont A (2004) Multicenter international study of oxaliplatin/5-fluorouracil/leucovorin in the adjuvant treatment of colon cancer (MOSAIC) Investigators: Oxaliplatin, fluorouracil, and leucovorin as adjuvant treatment for colon cancer. N Engl J Med 350:2343-2351

2. Lucchetta M, Lonardi S, Bergamo F, Alberti P, Velasco R, Argyriou AA, Briani C, Bruna J, Cazzaniga M, Cortinovis D, Cavaletti G, Kalofonos HP (2012) Incidence of atypical acute nerve hyperexcitability symptoms in oxaliplatin-treated patients with colorectal cancer. Cancer Chemo Pharm 70:899-902

3. Cersosimo RJ (2005) Oxaliplatin-associated neuropathy: a review. Ann Pharmacother 39:128-135

4. de Gramont A, Figer A, Seymour M, Homerin M, Hmissi A, Cassidy J, Boni C, Cortes-Funes H, Cervantes A, Freyer G, Papamichael D, Le Bail N, Louvet C, Hendler D, de Braud F, Wilson C, Morvan F, Bonetti A (2000) Leucovorin and fluorouracil with or without oxaliplatin as first-line treatment in advanced colorectal cancer. J Clin Oncol 18:2938-2947

5. Krishnan AV, Goldstein D, Friedlander M, Kiernan MC (2005) Oxaliplatin-induced neurotoxicity and the development of neuropathy. Muscle Nerve 32:51-60

6. Land SR, Kopec JA, Cecchini RS, Ganz PA, Wieand HS, Colangelo LH, Murphy K, Kuebler JP, Seay TE, Needles BM, Bearden JD 3rd, Colman LK, Lanier KS, Pajon ER Jr, Cella D, Smith RE, O'Connell MJ, Costantino JP, Wolmark N (2007) Neurotoxicity from oxaliplatin combined with weekly bolus fluorouracil and leucovorin as surgical adjuvant chemotherapy for stage II and III colon cancer: NSABP C-07. J Clin Oncol 25:2205-2211

7. Leonard GR, Wright MA, Quinn MG, Fioravanti S, Harold N, Schuler B, Thoomas RR, Grem JL (2005) Survey of oxaliplatinassociated neurotoxicity using an interview-based questionnaire in patients with metastatic colorectal cancer. BMC Cancer 5:116-126

8. Kaley TJ, Deangelis LM (2009) Therapy of chemotherapyinduced peripheral neuropathy. Br J Haematol 145:3-14

9. Cavaletti G, Alberti P, Marmiroli P (2011) Chemotherapyinduced peripheral neurotoxicity in the era of pharmacogenomics. Lancet Oncol 12:1151-1161

10. Wu Z, Ouyang J, He Z, Zhang S (2012) Infusion of calcium and magnesium for oxaliplatin-induced sensory neurotoxicity in colorectal cancer: a systematic review and meta-analysis. Eur J Cancer 48:1791-1798

11. Cavaletti G, Frigeni B, Lanzani F, Mattavelli L, Susani E, Alberti P, Cortinovis D, Bidoli P (2010) Chemotherapy-induced peripheral neurotoxicity assessment: a critical revision of the currently available tools. Eur J Cancer 46:479-494

12. Kono T, Kanematsu T, Kitajima M (2009) Exodus of Kampo, traditional Japanese medicine, from the complementary and alternative medicines: is it time yet? Surgery 146:837-840

13. Nagaki Y, Hayasaka S, Hayasaka Y, Kadoi C, Sekiya N, Terasawa K, Sakakibara I (2003) Effects of goshajinkigan on corneal sensitivity, superficial punctate keratopathy and tear secretion in patients with insulin-dependent diabetes mellitus. Am J Chin Med 31:103-109

14. Tawata M, Kurihara A, Nitta K, Iwase E, Gan N, Onaya T (1994) The effects of goshajinkigan, a herbal medicine, on subjective symptoms and vibratory threshold in patients with diabetic neuropathy. Diabetes Res Clin Pract 26:121-128

15. Uno T, Ohsawa I, Tokudome M, Sato Y (2005) Effects of Goshajinkigan on insulin resistance in patients with type 2 diabetes. Diabetes Res Clin Pract 69:129-135

16. Kono T, Mamiya N, Chisato N, Ebisawa Y, Yamazaki H, Watari J, Yamamoto Y, Suzuki S, Asama T, Kamiya K (2009) Efficacy of Goshajinkigan for peripheral neurotoxicity of oxaliplatin in patients with advanced or recurrent colorectal cancer. Evid Based Complement Alternat Med. doi:10.1093/ecam/nep200

17. Nishioka M, Shimada M, Kurita N, Iwata T, Morimoto S, Yoshikawa K, Higashijima J, Miyatani T, Kono T (2011) The Kampo medicine, Goshajinkigan, prevents neuropathy in patients treated by FOLFOX regimen. Int J Clin Oncol 16:322-327

18. Kono T, Mishima H, Shimada M, Morita S, Sakamoto J (2009) GONE Investigators: preventive effect of goshajinkigan on peripheral neurotoxicity of FOLFOX therapy: a placebo-controlled double-blind randomized phase II study (the GONE Study). Jpn J Clin Oncol 39:847-849 
19. Haller DG (2000) Safety of oxaliplatin in the treatment of colorectal cancer. Oncology (Williston Park) 12(Suppl 11):15-20

20. Omiya Y, Goto K, Suzuki Y, Ishige A, Komatsu Y (1999) Analgesia-producing mechanism of processed Aconiti tuber: role of dynorphin, an endogenous kappa-opioid ligand, in the rodent spinal cord. Jpn J Pharmacol 79:295-301

21. Gotoh A, Goto K, Sengoku A, Shirakawa T, Akao Y, Fujisawa M, Okada H, Arakawa S, Kamidono S (2004) Inhibition mechanism of Gosha-jinki-gan on the micturition reflex in rats. J Pharm Sci 96:115-123

22. Imamura $\mathrm{T}$, Ishizuka $\mathrm{O}$, Aizawa $\mathrm{N}$, Zhong $\mathrm{C}$, Ogawa $\mathrm{T}$, Nakayama T, Tanabe T, Nishizawa O (2008) Gosha-jinki-gan reduces transmitter proteins and sensory receptors associated with $\mathrm{C}$ fiber activation induced by acetic acid in rat urinary bladder. Neurourol Urodyn 27:832-837

23. Joseph EK, Chen X, Bogen O, Levine JD (2008) Oxaliplatin acts on IB4-positive nociceptors to induce an oxidative stress-dependent acute painful peripheral neuropathy. J Pain 9:463-472

24. Hu X, Sato J, Oshida Y, Xu M, Bajotto G, Sato Y (2003) Effect of Gosha-jinki-gan (Chinese herbal medicine: Niu-Che-Sen-QiWan) on insulin resistance in streptozotocin-induced diabetic rats. Diabetes Res Clin Pract 59:103-111
25. Ushio S, Egashira N, Sada H, Kawashiri T, Shirahama M, Masuguchi K, Oishi R (2012) Goshajinkigan reduces oxaliplatininduced peripheral neuropathy without affecting anti-tumour efficacy in rodents. Eur J Cancer 48:1407-1413

26. Tai T, Akita Y, Kinoshita K, Koyama K, Takahashi K, Watanabe K (1995) Anti-emetic principles of Poria cocos. Planta Med 61:527-530

27. Lee JH, Lee YJ, Kang SW, Kim Y, Shin M, Hong M, Seo EK, Kim SH, Nah SY, Bae H (2010) Effects of protostane-type triterpenoids on the 5-HT3A receptor-mediated ion current in Xenopus oocytes. Brain Res 1331:20-27

28. Cavaletti G, Marmiroli P (2010) Chemotherapy-induced peripheral neurotoxicity. Nat Rev Neurol 6:657-666

29. Durand JP, Deplanque G, Montheil V, Gornet JM, Scotte F, Mir O, Cessot A, Coriat R, Raymond E, Mitry E, Herait P, Yataghene Y, Goldwasser F (2012) Efficacy of venlafaxine for the prevention and relief of oxaliplatin-induced acute neurotoxicity: results of EFFOX, a randomized, double-blind, placebo-controlled phase III trial. Ann Oncol 23:200-205 